\title{
Le Web 2.0 peut-il être considéré comme un MOTIF ? Approche critique du concept à partir d'une relecture du texte de T. O'Reilly
}

Can Web 2.0 Be Seen in Terms of MOTIF? A Critical Approach to the Concept through a Political Analysis of T. O'Reilly's Work

\section{Athissingh Ramrajsingh}

\section{(2) OpenEdition}

Journals

Édition électronique

URL : http://journals.openedition.org/edc/2294

DOI : $10.4000 /$ edc. 2294

ISSN : 2101-0366

Éditeur

Université Lille-3

\section{Édition imprimée}

Date de publication : 1 décembre 2010

Pagination : 167-184

ISBN : 978-2-917562-04-8

ISSN : $1270-6841$

Référence électronique

Athissingh Ramrajsingh, « Le Web 2.0 peut-il être considéré comme un MOTIF ? Approche critique du concept à partir d'une relecture du texte de T. O'Reilly », Études de communication [En ligne], 35 | 2010, mis en ligne le 01 décembre 2012, consulté le 19 avril 2019. URL : http://journals.openedition.org/ edc/2294 ; DOl : 10.4000/edc.2294

Ce document a été généré automatiquement le 19 avril 2019

(C) Tous droits réservés 


\title{
Le Web 2.0 peut-il être considéré comme un MOTIF ? Approche critique du concept à partir d'une relecture du texte de T. O'Reilly
}

\author{
Can Web 2.0 Be Seen in Terms of MOTIF? A Critical Approach to the Concept \\ through a Political Analysis of T. O'Reilly's Work
}

Athissingh Ramrajsingh

1 Formalisé pour la première fois en 2005 par Tim O'Reilly dans un article (publié sur son blog) intitulé What is Web 2.0 ? (O'Reilly, 2005), le Web 2.0 a fait l'objet d'une production littéraire dense, aussi bien dans le monde « grand public » (Gervais, 2007 ; Fayon, 2008 ; Hussher F. X., Hussher C. et Carrasco, 2006) que dans le monde académique (Lefebvre, 2005 ; Morand, 2005 ; Rebillard, 2007) ${ }^{1}$. Nous proposons, dans cet article, de revenir sur ce texte fondateur, non pour donner une autre définition de ce qu'est le Web 2.0, mais pour en avoir une lecture politique ${ }^{2}$.

2 En effet, depuis 2005, de nombreux articles, ouvrages ou blogs font référence au Web 2.0, voire même tentent d'en donner des applications concrètes pour les entreprises ${ }^{3}$. Sans pour autant remettre en question ces apports, il nous paraît toutefois primordial de revenir au texte fondateur qui, paradoxalement, reste peu étudié - alors qu'il s'agit de la première formalisation concrète de ce terme - et qui pourtant nous semble un objet d'étude aussi important que les applications qui peuvent en découler.

3 Ce " texte objet ", dont nous proposons une analyse, peut être appréhendé de différentes manières à l'image ce que Jeanneret nomme une représentation circulante de la divulgation (Jeanneret, 2008) ou bien grâce à des cadres théoriques issus aussi bien de la sémiotique, de la linguistique, voire de la sociologie des techniques (Proulx, MassitFolléa et Conein, 2009).

4 Or, ce texte nous apparaît également fortement politique. C'est la raison pour laquelle nous avons choisi de nous focaliser, non sur une analyse à partir des cadres 
préalablement cités, mais plutôt à partir d'un concept de P. Robert, le MOTIF (MOTeur d'Inférences et de gestion des Formes) que nous présenterons en détail dans la première partie de l'article.

5 Le choix de ce positionnement, volontairement politique, n'est pas anodin. En effet, certains des blogueurs Nord-américains les plus lus, par ailleurs très critiques sur le Web 2.0 se placent sur ce registre : Jaron Lanier parle de maoïsme digital, Nicholas Carr d'amoralisme du Web et Andrew Keen de culte de l'amateur.

Sans pour autant adopter une démarche idéologique, à l'instar de ces auteurs, ces attaques révèlent plus ou moins explicitement une critique politique du Web 2.0, raison pour laquelle nous proposons cette lecture.

De ce fait, après avoir vu les fondements théoriques de notre démarche qui s'appuie sur le MOTIF (1), nous confronterons ce cadre aux différents points développés par T. O'Reilly dans son article (2) avant de voir, dans un dernier temps, en quoi nous pouvons avoir une lecture politique de ce qu'est le Web 2.0 (3).

\section{Le MOTIF : Le MOTeur d'Inférence et de Gestion des Formes}

\section{Le MOTIF}

Le MOTIF (Robert, 2000) ${ }^{4}$ s'appuie sur un concept fondamental développé par un économiste, L. Thévenot, les investissements de formes (Thévenot, 1985). Pour Thévenot, au-delà des machines, des matières premières et des personnels, l'entreprise fonctionne également grâce à la " mise en cuvre de tout un ensemble 'd'outils' complémentaires, normes, standards, règlements, conventions, contrats, qualifications, marques etc [...] » (Thévenot, 1985, p. 22). Et comme l'indique P. Robert, L. Thévenot fait par trois fois référence à l'informatique (aux pages 39,40 et 50 ) et soutient que :

[...] les technologies informatiques, après avoir impliqué, dans une première période de leur développement un codage des informations traitées, favorisent dans une deuxième étape l'application d'un codage beaucoup plus général des formes gérées par l'entreprise (Thévenot, 1985, p. 50).

Cette idée directrice, P. Robert la résume ainsi :

" L. Thévenot mêle codes/contraintes et équipements, or, l'informatique constitue par excellence une technologie qui croise inextricablement ces trois composantes: elle se concrétise effectivement par/dans des machines, gérées par et gestionnaires de codes, produisant de réelles contraintes [...] " ajoutant que l'informatique reste un investissement machinique de forme autorisant la gestion du nombre (Robert, 2000).

Les principes du MOTIF peuvent être ainsi résumés :

- L'informatique, dans sa dimension " machine ", est un moteur d'inférence et de gestion des formes.

- L'informatique est une variable permettant de produire des formes, à condition d'être elle-même contrôlée. La maîtrise de la production de flux et de formes internes permet de produire des flux et des formes externes.

- Les interfaces améliorées participent à faire baisser la vigilance de l'utilisateur visà-vis de la machine.

- Le travail des formes repose sur des logiques de standardisation/normalisation.

11 La définition des formes ainsi que leur relation avec les inférences sont également précisées : 
La forme, c'est ce travail, ce mouvement, de l'un, le moule, à l'autre, l'objet. Pourquoi un mouvement ? Parce que la forme n'est pas stricto sensu seulement dans le moule ou seulement autour de l'objet, mais bien leur relation même, leur mise en relation : application du moule (en tant que frontières et structures) sur ce qu'il in-forme, met en forme (matières ou idées). Une relation que l'on peut qualifier d'inférence (ibid.).

12 Cette approche des flux et des formes présentée par P. Robert, inspirée par L. Thévenot se traduit entre autres, au travers du Web 2.0, dans les données ${ }^{5}$, aspect essentiel sur lequel nous nous attarderons.

\section{Les propriétés du MOTIF}

D'un point de vue sociologique, le MOTIF met ainsi en lumière trois points cruciaux :

- Premièrement, la gestion du multimédia et des interconnexions (en d'autres termes, les formes produites par le MOTIF et la gestion de celles-ci) se traduit par rapport à deux plans : réversibilité

généralisée ${ }^{6}$ au niveau local et irréversibilité ${ }^{7}$ au niveau global. Ces deux éléments ne sont pas formulés en tant que finalités, mais en tant que craintes.

- Deuxièmement, la gestion des hommes est " facilitée ${ }^{8}$. Car le MOTIF, en tant que producteur de formes, non seulement permet l'enregistrement de données (formes), mais offre, par la même occasion, la possibilité de gérer ces dernières. Les nouvelles technologies Web ainsi que la gestion de l'identité numérique en sont des exemples révélateurs. Par ailleurs, P. Robert précise " [qu'] il ne faut pas se cacher qu'Internet offre également de considérables ressources nouvelles, en matière de traçabilité des activités et des goûts, qui, elles, peuvent être directement mobilisées au profit des logiques étatique et marketing !» (Robert, 2000, op. cit.).

- Troisièmement, la gestion des processus est rendue plus efficace. En effet, le nombre (de personnes et de documents) n'est plus un obstacle. Plus exactement, les limites posées par le nombre sont repoussées : « Le MOTIF permet de mieux et surtout de plus facilement maitriser ce nombre [...] ; Il rend possible l'articulation entre la multiplication des classes et celle des croisements " (ibid.).

14 Afin de percevoir la cohérence de cette interprétation dans sa globalité, nous allons reprendre les principes posés par Tim O'Reilly dans son article "What is Web 2.0 » (O’Reilly, 2005) pour montrer comment chaque point peut être explicité à partir du MOTIF.

\section{Le Web 2.0 d'O'Reilly, un MOTIF ?}

Cette remise en perspective suppose alors de reconsidérer le titre lui-même : " Qu'est-ce que le Web 2.0 : modèles de conception et d'affaires pour la prochaine génération de logiciels ${ }^{9}$.

De ce titre se dégage une orientation à la fois économique (modèles d'affaires) et technique (modèles de conception). Le propos est donc clairement annoncé : comment concevoir des logiciels (mais aussi des services) dans le cadre de la mutation du Web, tout en adaptant les modèles économiques ? L'introduction va d'ailleurs dans ce sens, et même jusqu'à comparer le Web 2.0 à une révolution industrielle :

[...] le fait qu'une bulle se forme puis éclate est un trait commun à toutes les révolutions industrielles [...]. De plus, les quelques sociétés qui avaient survécu à l'hécatombe [de la bulle Internet de 2001] semblaient avoir quelque chose de commun. Se pouvait-il que le crash des " dot com " eût révélé une nouvelle ère pour le Web au point que l'expression « Web $2.0 »$ ait un sens ? [OR $]^{10}$. 
17 Le titre ne fait nullement mention des usages. Le seul terme employé est celui de « modèles d'affaires ". De ce point de vue, le Web 2.0 est un modèle économique reposant sur certains principes, notamment techniques. Autrement dit, le Web 2.0 pourrait apparaître comme la réponse à la question suivante : " Comment gagner de l'argent avec les nouvelles technologies Web ?». L'apport théorique qu'aurait pu représenter cet article s'efface alors derrière une sorte de " mode d'emploi » - qu'il semble être devenu - afin d'orienter la stratégie des entrepreneurs et financiers.

Cette première analyse critique du titre nous permet d'aborder le texte d'o'Reilly au travers du MOTIF. Nous tenterons ensuite de comprendre comment, au niveau du discours, le Web 2.0 concentre un certain nombre de propriétés du MOTIF, avant d'en déduire une lecture politique.

\section{Le Web en tant que plate-forme : la décentralisation individuelle au service de la centralisation collective}

19 Premier pilier du Web 2.0, ce principe du « Web en tant que plate-forme » érige le Web, non comme un « service parmi d'autres » fourni sur Internet, mais comme une véritable plate-forme. Mais que sous-entend cette approche ?:

À la base, Google nécessitait une compétence dont Netscape n'avait jamais eu besoin : la gestion de bases de données. Google n'est pas une simple suite d'outils logiciels, c'est une base de données spécialisée. Sans données, les outils ne servent à rien ; sans logiciels, les données sont ingérables [...]. En fait, la valeur d'un logiciel est proportionnelle à l'échelle et au dynamisme des données qu'il permet de gérer [OR]. d'utilisateurs permet la création, mais aussi la sélection, d'un contenu plus pertinent. L'effet de " foule " agit ainsi, toujours pour O’Reilly, comme un filtre décentralisé et distribué, renforçant ainsi son efficacité.

\section{L'intelligence collective : s'appuyer sur le bénévolat à des fins économiques}

L'auteur présente ensuite une longue série d'innovations destinées à illustrer la manière dont certains acteurs marchands, tel Amazon, ont non seulement mis en place des outils d'orientation pour les consommateurs (meilleures ventes, les internautes ayant consulté ce produit ont également consulté tels autres...), mais également encouragé l'implication de ces 
derniers, en vue d'augmenter leur chiffre d'affaires. En somme, semble-t-il dire, une information pertinente favorise les achats.

Cependant, à aucun moment l'auteur ne fait référence à un élément dont l'importance est pourtant capitale : l'augmentation croissante et exponentielle de la masse d'informations disponibles, qu'il s'agisse de documents ou de données personnelles.

Autrement dit, plus un acteur est capable de " faire travailler les internautes » dans un cadre répondant à leurs attentes, plus le retour sur investissement sera conséquent :

S'opère ainsi une mise en marché de l'expression individuelle des internautes ordinaires, comme si elle constituait une niche commerciale pour les nouveaux services culturels sur mesure promus par les acteurs de l'industrie des NTIC (Allard et Vandenberghe, 2003).

En somme, la capacité des acteurs importants à générer des flux et à récupérer des formes conçues à l'origine par les internautes pour se les approprier rejoint, selon O'Reilly, des problématiques économiques de premier plan.

\section{La puissance est dans les données, ou le renforcement du pouvoir par l'information}

Pour O'Reilly, les services en tant que tels ne constituent plus un marché rentable, dans la mesure où ils sont le plus souvent proposés à titre gratuit :

La course pour la possession de données stratégiques a déjà commencé [...]. Souvent, le gagnant sera la société qui atteindra la première une masse critique de données par agrégation des utilisateurs et convertira cet avantage en services [OR].

Pour ces acteurs, il s'agit donc bien d'une course : pour le contrôle des informations, l'utilisation des services, l'amélioration et l'enrichissement des données... Ne peut-on pas alors y déceler une volonté politique ? Rappelons qu'historiquement, seuls les États avaient la puissance nécessaire pour collecter, gérer et stocker la masse d'informations concernant leurs administrés, notamment par le biais du recensement. Cette opération, sous prétexte de connaître la situation de la population, servait autrefois à calculer la gabelle $^{12}$. Puis, à partir de 1801, Napoléon a instauré le recensement général tel que nous le connaissons actuellement, afin de mettre en place la répartition du paiement de l'impôt. Aujourd'hui, cette mission est dévolue à l'INSEE, à qui l'État français fournit, de manière exclusive, les moyens nécessaires pour la mener à bien. Ainsi, la légitimité conférée par le pouvoir donne à l'État le droit de connaitre certains aspects de la vie des citoyens (afin, par exemple, d'évaluer le paiement de l'impôt). En revanche, le monde marchand doit « construire » cette légitimité vis-à-vis des consommateurs. Il doit donc la gagner en fournissant des services susceptibles de trouver une clientèle potentielle. Plus ceux-ci seront utilisés, plus ils seront connus et, par voie de conséquence, gagneront en notoriété. Et comme nous l'avons déjà démontré (Ramrajsingh, 2009), sur le Web, la notoriété conduit à la légitimité. Une fois celle-ci acquise et renforcée par l'instauration, entre autres, de mécanismes de confiance, ils pourront mettre en place leurs propres dispositifs de collecte. Ils auront alors édifié les fondements de leur modèle d'affaires, qui s'appuiera notamment sur la qualité des liens hypertextes, tout en améliorant continuellement leurs services. 


\section{La fin des cycles de release, ou l'expérience utilisateur transparente au service de l'opacité}

Le quatrième point d'argumentation concerne donc les cycles :

Cal Henderson, le principal développeur de Flickr, a révélé récemment qu'il déployait une nouvelle version de son application toutes les demi-heures [...]. Le business model de Microsoft dépend de la remise à niveau que chacun fait sur son PC tous les deux ou trois ans. Celui de Google ne dépend que de la capacité des utilisateurs à se servir des nouvelles fonctionnalités qui leur sont proposées [OR].

L'époque où la mise à jour des logiciels ou des services s'effectuait de manière ponctuelle semble donc révolue pour l'auteur. Aujourd'hui, le rythme est continu, sans que l'utilisateur n'en soit informé.

31 De manière paradoxale donc, la fin des cycles sonne l'avènement de la technique. Cette invisibilité des évolutions pour l'utilisateur revêt un caractère irréversible ${ }^{13}$, dès lors qu'il est impossible de revenir à une version antérieure. Par rapport à une démarche qui se voudrait « libertaire ", nous sommes donc en présence d'un mouvement inverse. En effet, l'opacité renforce le sentiment de " boîte noire » (Vedel, 2003) et favorise ainsi le pouvoir de contrôle (Robert, 2005).

La fin des cycles logiciels induit ainsi une concentration technique de plus en plus importante. À celle-ci s'ajoute un renforcement des moyens de gestion qui, en obligeant l'internaute à utiliser une version précise de tel ou tel service, le rendent totalement dépendant de certaines prises de position techniques (ou juridiques, comme la modification unilatérale des conditions d'utilisation), avec toutes les conséquences qui en découlent.

\section{Des modèles de programmation légers, ou comment favoriser la diffusion et l'appropriation pour renforcer sa présence}

Argument phare du Web 2.0, sa " simplicité » d'utilisation (pour l'internaute) et de programmation (pour les développeurs), qui se traduit par une miniaturisation de l'équipement et une simplification du code (au sens où, pour faire un lien hypertexte dans une page, par exemple, il n'est plus nécessaire de connaître la syntaxe HTML exacte).

Or, concevoir des programmes légers (ou des interfaces simplificatrices) ne va pas sans en affecter la compréhension par le plus grand nombre. Car optimiser le code implique de posséder des compétences techniques pointues. Celui-ci devient alors une nouvelle source de pouvoir pour les experts, non par la création d'irréversibilités, comme sur les platesformes de services, mais par le détournement de son utilisation initiale. L'article le confirme d'ailleurs implicitement : "L'interface de Google Maps, bâtie sur les principes d'AJAX, a rapidement été décryptée par quelques hackers, qui ont alors utilisé ces données pour bâtir de nouveaux services " [OR].

La solution préconisée revêt donc un caractère radical : d'une part, il faudrait optimiser le code en le rendant plus léger ; d'autre part, il faudrait simplifier son accès afin qu'il soit plus facilement réutilisable, en gardant à l'esprit le facteur de décentralisation : « Pensez syndication, pas coordination »[OR]. 
L'information déjà abondante nécessite des outils de gestion. En s'appuyant sur l'intelligence collective ainsi que sur la capacité des " experts ", les acteurs majeurs trouvent ainsi, dans un contexte de multiplication des données, des opportunités de marché à moindre frais :

Nous pensons que le Web 2.0 apportera des opportunités pour des sociétés d'entrer dans la compétition simplement en intégrant et en assemblant des services fournis par d'autres [OR].

Le principe économique du Web 2.0 suit, à nouveau, une logique paradoxale. En effet, il se fonde sur la réappropriation d'applications ayant été créées par d'autres avec, toujours en parallèle, l'explosion informationnelle. Or, les acteurs pouvant traiter cette masse d'informations ne sont pas légion. Le phénomène de concentration qui en résulte est alors comparable à ce que l'on retrouve dans les médias, à un degré de plus en plus élevé (Meier, 2005).

\section{Le logiciel se libère du PC, ou comment restreindre la liberté individuelle de l'internaute}

Autre idée phare du Web 2.0 développée par O’Reilly, cette nouvelle approche de la décentralisation complète l'idée du Web en tant que plate-forme :

[...] l'aspect « 2.0 » n'est pas dans la nouveauté, mais plutôt dans la pleine réalisation du véritable potentiel de la plate-forme Web, celle-ci devant nous guider pour comprendre comment concevoir applications et services [OR].

Tout d'abord, les modèles de programmation vont être de plus en plus allégés, afin d'une part, d'être exploitables par les ordinateurs, et d'autre part, d'être en adéquation avec le débit permis pour l'internaute (outils de gestion des flux). Ensuite, le pouvoir des données va être renforcé. Dans cette optique, chacun pourra déposer ses documents sur un espace distant (la plate-forme) et virtuellement accessible depuis n'importe quel ordinateur disposant d'un accès au réseau ${ }^{14}$. Cette masse de documents stockés deviendra l'enjeu d'une nouvelle guerre, laquelle d'ailleurs a déjà commencé (création de formes). De plus, la multiplication des données et des documents créera un nouveau besoin en outils de gestion de ces derniers (nécessité de gérer les formes). L'internaute deviendra alors complètement tributaire, non seulement de l'accès au réseau, mais également de l'accès aux services en question. Car en cas d'indisponibilité de ces derniers, il lui faudra trouver un recours (gestion des flux et des formes) :

À ce jour, iTunes est le meilleur exemple de ce principe. Cette application va sans cesse d'un appareil portable à un système Web massif, le PC servant uniquement de mémoire locale et de station de contrôle [OR].

En prenant comme exemple Apple et sa plate-forme de téléchargement de musique, l'auteur tente d'étayer sa thèse, et ce à deux niveaux. D'une part, il montre de quelle manière l'outil Web, en tant que plate-forme, peut héberger un service. D'autre part, il explique comment l'utilisation d'un appareil portable (en l'occurrence l'IPod) peut transformer un PC non plus en terminal final, mais en station de contrôle relais pour d'autres appareils, les logiciels étant installés d'un côté sur le Web et de l'autre dans l'IPod - Cette intégration totale, gérée dans ce cas précis par Apple, illustre le leurre de la décentralisation évoqué précédemment : sous couvert d'une plus grande mobilité pour l'utilisateur (il s'agit ici de pouvoir écouter - mais surtout acheter - de la musique en 
tout lieu), ce dernier devient dépendant d'un unique acteur marchand ayant réussi à faire converger plusieurs technologies.

De surcroît, l'utilisateur n'a plus aucune possibilité de contrôler ses flux et formes, ne serait-ce qu'à son échelle. Car cette fonction est à présent dévolue aux outils hébergés " à distance ".

Enfin, l'auteur termine par un dernier point qui, s'il peut sembler secondaire, se révèle un moyen concret d'application des principes énoncés : il s'agit de l'enrichissement de l'interface des utilisateurs.

\section{Enrichir les interfaces utilisateurs : « le beau » qui masque les enjeux}

Le potentiel de richesse d'interface du Web ne fut jamais exploité par les grandes applications, jusqu'à ce que Google lance Gmail, rapidement suivi par Google Maps : des applications Web possédant un niveau d'interactivité équivalent à un logiciel PC classique [OR] ${ }^{15}$.

Les interfaces sont aujourd'hui beaucoup plus épurées qu'à l'origine de l'Internet grand public. La page d'accueil du moteur de recherche Google étant l'une des illustrations les plus symboliques.

Depuis, un certain nombre de langages (Flash) ou des combinaisons de technologies existantes (AJAX) ont permis de revenir sur cet aspect essentiel, véritable lien entre l'internaute et le service.

Mais ces interfaces ne se contentent pas d'être agréables visuellement. À leur " richesse " s'ajoute également un caractère très fonctionnel, à tel point que certains internautes sont amenés à les considérer comme de véritables guides, ce qui peut parfois aller jusqu'à l'extrême, prenant en exemple le carnet d'adresses :

Un carnet d'adresses Web 2.0 pourra d'une part traiter un ensemble de contacts mémorisés comme le fait par exemple un téléphone. Parallèlement à cela, un agent de synchronisation Web, dans le style de Gmail, se souviendra de chaque message envoyé ou reçu, de chaque adresse e-mail ou numéro de téléphone utilisé et s'appuiera sur des heuristiques de réseaux sociaux pour décider quelles alternatives offrir en cas de recherches infructueuses [OR].

Ainsi, selon l'auteur, des interfaces riches associées à des agents intelligents assureront à l'internaute une réelle assistance au niveau de sa recherche de contacts. Ces nouvelles applications pourront s'appuyer, d'une part, sur des phénomènes de convergence technique et de partage des standards, et, d'autre part, sur les immenses capacités de stockage et de traitement des données des acteurs marchands. Or, quelle est la vocation de ces interfaces riches ? Ne consiste-t-elle pas, entre autres, à proposer l'accès à des données que l'utilisateur aura lui-même fournies au préalable?

Les interfaces riches ne doivent donc pas être considérées seulement comme plus belles ou plus agréables. Elles occupent une véritable fonction d'assujettissement, d'autant plus insidieusement qu'elles sont invisibles aux yeux de l'internaute. Celui-ci n'y verra d'ailleurs que des avantages, tant en termes d'accessibilité que de convivialité. À aucun moment il ne lui viendra à l'esprit que l'utilisation de ces services, associée à la divulgation d'informations personnelles, participe pleinement à renforcer le pouvoir économique de ces acteurs marchands. Et sa vigilance vis-à-vis de la machine en devient d'autant moins forte, puisque les services sont plus fonctionnels pour lui. 
également aux données :

L'opportunité pour de nouveaux arrivants d'exploiter pleinement le potentiel du Web 2.0 entraînera la création d'applications qui apprendront de leurs utilisateurs en s'appuyant sur une architecture de participation et qui se démarqueront non seulement par leur interface, mais aussi par la richesse des données partagées qu'elles offriront [OR].

49

Lauteur, de façon très implicite, ne semble pas aborder la question autrement : les interfaces sont tenues d'être riches et d'exploiter un certain nombre de données, qui doivent certes profiter aux utilisateurs, mais tout " en s'appuyant sur eux ». En somme, il faut tirer parti de l'intelligence collective pour capitaliser de l'information, dans un processus d'irréversibilité au niveau global, mais créateur de conditions de réversibilité au niveau local.

En résumé, nous pouvons reprendre les propos de Jonckheere et Schreuer qui notent que " À bien des égards, le blabla technologique acritique entourant ce Web 2.0 joue un rôle de propagande au service de ce modèle économique bien particulier " (Jonckheere et Schreuer, 2008).

\section{Le Web 2.0 concept politique ?}

51 Nous nous sommes déjà largement attardés sur les éléments évoqués par O’Reilly, avant de les confronter aux particularités du MOTIF, tel que P. Robert le définit. Il s'agit maintenant de traiter la question des propriétés, en particulier au niveau des réversibilités. Car si au niveau micro, des réversibilités demeurent encore possibles, c'est oublier l'irréversibilité au niveau macro. Ces illustrations répondent en partie à la question posée par Pascal Robert :

Ne peut-on craindre, assez paradoxalement, que la réversibilité généralisée au niveau " local » ne se paye d'une irréversibilité elle-même généralisée au niveau « global »?(Robert, 2000, op. cit.).

Si cette irréversibilité est perceptible au niveau des contenus, elle l'est tout autant dans la gestion des hommes comme nous l'avons déjà évoqué. Ce qui n'est pas sans rappeler la notion de panoptique, telle du moins qu'elle est redéfinie par Deleuze :

La formule abstraite du Panoptisme n'est plus « voir sans être vu ", mais imposer une conduite quelconque à une multiplicité humaine quelconque (Deleuze, 2004, p. 41).

Cette conduite imposée rejoint le caractère irréversible du Web 2.0 en tant que MOTIF, en favorisant fortement l'accès à la création de contenus, mais également à la mise en ligne de l'identité de chaque utilisateur. Et ce, en générant les conditions nécessaires pour créer ce panoptique, qui rejoint ainsi la question de la non neutralité de la technique.

C'est en ce sens que le discours autour du Web 2.0, formulé par T. O'Reilly apparaît comme une véritable opération politique dans la mesure où certains acteurs privés remplissent des fonctions historiquement politiques. Autrement dit, le Web 2.0 apparait aussi bien comme forme panoptique, qu'en tant qu'outil d'une forme de gouvernement par l'économique (ou outil qui permet de nouer l'économique au politique avec un déplacement du pouvoir, des gouvernants légitimes aux marchands). 


\section{BIBLIOGRAPHIE}

Allard, L., Vandenberghe, F., (2003), Express yourself ! Les pages perso. Entre légitimation technopolitique de l'individualisme expressif et authenticité réflexive peer to peer, in Réseaux, $\mathrm{n}^{\circ} 117$, pp. 191-219.

Beniger, J. R., (1986), The Control Revolution : Technological and Economic Origins of the Information Society, Cambridge, MA, Harvard University Press, 508 p.

Carr, N., (2005), The amorality of Web 2.0, in Rough Type : Nicholas Carr's blog : http:// roughtype.com/archives/2005/10/the_amorality_o.php, date de la dernière visite :

17 Mars 2010.

Deleuze, G., (2004-1986), Foucault, Paris, Éditions de Minuit, 143 p.

Després-Lonnet, M. et Cotte, D., (2007), Nouvelles formes éditoriales en ligne, in Communication et langages, $\mathrm{n}^{\circ}$ 154, pp. 111-122.

Ellul, J., (2004-1977), Le système technicien, Paris, Le cherche midi, 337 p.

Ellul, J., (1988), Le bluff technologique, Paris, Hachette, 748 p.

Fayon, D., (2008), Web 2.0 et au-delà - Nouveaux internautes : du surfeur à l'acteur, Paris, Économica, $224 \mathrm{p}$.

Gensollen, M., (2001), Internet : Marché électronique ou réseaux commerciaux ?, in Revue économique, vol. 52, numéro hors série, octobre 2001, pp. 137-161.

Gervais, J.-F., (2007), Web 2.0 : Les internautes au pouvoir, Paris, Dunod, 216 p.

Jonckheere, C. et Schreuer, F., (2008), Pour une critique politique du Web 2.0, in Politique, $\mathrm{n}^{\circ} 54$ : http://politique.eu.org/archives/2008/04/682.html, date de la dernière visite :5 Août 2009.

Huet, J. M. et Tcheng, H., (2008), Web 2.0 et utopie : faut-il brûler Wikipédia ?, in Journal du Net : http://www.journaldunet.com/expert/27024/web-2-0-et-utopie-faut-il-bruler-wikipedia.shtml, date de la dernière visite : 6 Juin 2009.

Hussher, F.-X., Hussher, C. et Carrasco, M.-E., (2006), Le nouveau pouvoir des internautes, Paris, Timée-Éditions, $320 \mathrm{p}$.

Keen, A., (2007), The Cult of the Amateur : How Today's Internet is Killing Our Culture, NY, Crown Business, 240 p.

Lanier, J., (2006), Digital Maoism : the Hazards of the New Online Collectivism, in Edge.org : http:// edge.org/3rd_culture/lanier06/lanier06_index.html, date de la dernière visite :22 Février 2010. Lefebvre, A., (2005), Les réseaux sociaux : pivot de l'Internet 2.0, Paris, M2 Éditions, 200 p. Lévy, P., (2002), Cyberdémocratie : essai de philosophie politique, Paris, Odile Jacob, 283 p. Meier Werner, A., (2005), Media concentration gouvernance : une nouvelle plate-forme pour débattre des risques ?, in Réseaux, $\mathrm{n}^{\circ}$ 131, pp. 17-52.

Morand, J.-C. et al., (2005), RSS, blogs : un nouvel outil pour le management, Paris, M2 Éditions, 295 p. 
Proulx, S., Massit-Follea, F. et Conein, B. (éds.), (2005), Internet, une utopie limitée. Nouvelles régulations, nouvelles solidarités, Laval, Presses de l'Université Laval, 335 p.

Ramonet, I., (2004), Le nouvel ordre Internet, in Le Monde Diplomatique : http://www.mondediplomatique.fr/2004/01/RAMONET/10615, date de la dernière visite :19 Juillet 2009.

Rebillard, F., (2006a), Du traitement de l'information à son retraitement - La publication de l'information journalistique sur l'internet, in Réseaux, $\mathrm{n}^{\circ}$ 137, pp. 29-68.

Rebillard, F., (2006b), L'externalisation des activités de création dans les industries de la culture, de l'information, et de la communication. A propos de tendances observables dans le secteur de la presse, in Actes du colloque international Mutations des ICIC, Observatoire des mutations des industries culturelles.

Rebillard, F., (2007), Le web 2.0 en perspective, Paris, L'Harmattan, 162 p.

Robert, P., (1994), L'impensé informatique, au miroir du quotidien Le Monde (1972-1980), Thèse en sociologie des techniques, sous la direction de P. Breton, Université Paris I - PanthéonSorbonne, $675 \mathrm{p}$.

Robert, P., (1996a), Le paradoxe de la simultanéité, hypothèse sur le cadre d'interprétation de l'histoire des technologies de l'information et de la communication (XIX ${ }^{e}-X^{e}$ siècle), in Médiaspouvoirs, $\mathrm{n}^{\circ} 42$, pp. 195-204.

Robert, P., (1996b), Technologies de l'information sans frontières ? La fonction frontière à l'épreuve des technologies de l'information et de la communication, in Technologies de l'Information et Société, $\mathrm{n}$

- 8 (3), pp. 213-242.

Robert, P., (2004), Le modèle CRITIC :contribution à l'élaboration d'une théorie macro-sociétale critique de l'informatique en sciences de l'information et de la communication, Mémoire d'habilitation à diriger des recherches, Université Paris 4 - Sorbonne, 367 p.

Robert, P., (2005), La logique politique des technologies de l'information et de la communication, Bordeaux, Presses universitaires de Bordeaux, $308 \mathrm{p}$.

Robert, P., (2000), Le MOTIF : de l'informatique comme moteur d'inférence et de gestion de formes, in Solaris, $\mathrm{n}^{\circ} 7$, Matière numérique : la production et l'invention des formes : Vers une esthétique nouvelle, sous la direction d'Odile Blin : http://biblio-fr.info.unicaen.fr/bnum/jelec/Solaris/ d07/7robert.html, date de la dernière visite :27 Septembre 2009.

Thévenot, L., (1985), Les investissements de forme, in Cahiers d'étude de l'emploi, n 29, pp. 21-71. Vedel, T., (2003), L'idée de démocratie politique : origines, visions, questions, in Le désenchantement démocratique, Perrineau Pascal (dir.), La Tour d'Aigues, Éditions de l'Aube, pp. 243-266.

Vitalis, A., (2002), La vie privée entre protection et exhibition, in Baudry, Sorbets et Vitalis (dirs.), La vie privée à l'heure des médias, Bordeaux, Presses universitaires de Bordeaux, pp. 185-197.

\section{NOTES}

1. Les ouvrages et articles sur le Web 2.0 sont très nombreux, aussi bien dans la littérature francophone qu'anglo-saxone. Nous choisissons volontairement de ne faire référence qu'à quelques travaux francophones.

2. Bien qu'il ne s'agisse pas d'un texte de « recherche », le statut d'o'Reilly, éditeur influent dans le monde informatique, a donné à ce texte une portée d'une ampleur considérable, au point d'en avoir popularisé l'expression. 
3. Voir par exemple le livre blanc de Digimind sur l'utilisation des outils Web 2.0 appliqués à la veille.

4. Cet article a été publié dans une revue électronique, Solaris. Il n’y a donc pas de numéro de page.

5. Il s'agit ici d'illustrer la notion de « forme » au travers des données tout en considérant qu'il ne s'agit que d'une déclinaison possible et non la seule.

6. La notion de réversibilité signifie « qu'un même support physique est susceptible d'exprimer plusieurs formes logicielles. Dès lors la machine multimédia est d'abord multiforme: le MOTIF peut en ce sens inférer et gérer une multiplicité de formes autrefois concrétisées dans des outils séparés... » (Robert, 2000).

7. La notion d'irréversibilité, quant à elle, renvoie à l'autonomie du système technicien de J. Ellul : "Ce retour direct du MOTIF sur lui-même, cette gestion des interconnexions entre ses multiples avatars, toujours dépendante de ce même MOTIF, n'ouvre-t-elle pas la perspective d'une irréversibilité triomphante, celle de l'autonomie du système technique, telle que l'avait envisagée voilà déjà plus de 20 ans un J. Ellul (1977) ?» (Robert, 2000).

8. La gestion des hommes, prérogative historiquement politique est aujourd'hui captée par des intérêts privés: "Depuis l'essor de la première mécanographie d'H. Hollerith à la fin du XIXe siècle jusqu'à aujourd'hui, le MOTIF s'articule directement au développement des méthodes statistiques de comptage et de classement du nombre, c'est-à-dire du grand nombre des hommes que se doivent de gérer nos démocraties complexes. La démarche touche aussi bien au politique, à travers l'opération fondamentale $d u$ recensement et la mise en place d'un outil statistique considérable au service de l'État-providence, qu'à l'économique, avec l'émergence et, désormais, l'imposition d'une logique marketing qui cherche à affiner la connaissance de ses marchés " (Robert, 2000).

9. Toutes les références à l'article de Tim O’Reilly publié en 2005 seront notées [OR]. L'article étant publié sur un blog, il n'y a pas de numéro de page.

10. Les références à l'article fondateur de T. O'Reilly seront notées [OR] à la suite de la citation.

11. Nous faisons ici référence à Google, puisque l'auteur y fait référence mais d'autres exemples existent tels que Facebook, ou Apple (avec ITunes).

12. Taxe sur le sel.

13. La création d'irréversibilités est une autre caractéristique du MOTIF.

14. Nous pensons notamment à GoogleDocs.

15. O’Reilly, 2005.

\section{RÉSUMÉS}

Qu'est-ce que le Web 2.0 ? A cette question, difficile, cet article tente d'apporter une réponse, modeste, mais s'appuyant sur une théorie, le MOTIF, développée par Pascal Robert. Ce Moteur d'Inférences et de gestion des Formes est un cadre d'analyse présenté comme permettant d'esquisser une théorie sociétale de l'informatique. A travers une relecture de l'article de Tim O'Reilly, What is Web 2.0 ? (publié le 30 Septembre 2005 sur son blog), nous tentons de comprendre en quoi le Web 2.0, plus qu'un changement paradigmatique en termes techniques, de pratiques ou économiques, se doit d'être interrogé au travers d'un questionnement politique, en particulier au niveau des enjeux de pouvoir sur la Toile. L'utilisation du MOTIF permet cette 
lecture en mêlant des considérations économiques, techniques et sociétales dans une perspective politique.

What is Web 2.0? This paper answers this question by using Pascal Robert's conceptualization of information technology as "MOTIF," that is, as a mechanism for inference and the management of forms. The paper proposes a political reading of Tim O'Reilly's 2005 text What Is Web 2.0? By using the MOTIF approach, we show how issues of power play out on the Web.

\section{INDEX}

Keywords : web 2.0, IT economy, IT political approach, social representations, IT criticism Mots-clés : web 2.0, approche critique des TIC, économie des TIC, représentations sociales, approche politique des TIC

\section{AUTEUR}

\section{ATHISSINGH RAMRAJSINGH}

CHERPA - Institut d'Etudes Politiques d'Aix-en-Provence Athissingh Ramrajsingh est post-doctorant rattaché au CHERPA (Croyance, Histoire, Espace, Régulation Politique et Administrative), EA 4261 de l'Institut d'Études Politiques d'Aix-enProvence, l'auteur a soutenu une thèse en 2009 intitulée « Les nouvelles technologies Web, facteur d'un glissement de la prérogative politique? » sous la direction de Pascal Robert. Ses recherches portent sur les enjeux politiques des TIC, ainsi que sur des aspects critiques de la " gestionarisation » de la société. Adresse électronique : athissingh.ramrajsingh@sciencespoaix.fr. 